\title{
miR-194-5p protects against myocardial ischemia/reperfusion injury via MAPK1/PTEN/AKT pathway
}

\author{
Qiufeng Zhang ${ }^{1}$, Xiaotian $\mathrm{Wu}^{1}$, Jie Yang ${ }^{2}$ \\ ${ }^{1}$ Emergency Department, First People's Hospital of Shangqiu City, Shangqiu, China; ${ }^{2}$ Department of Cardiovascular Medicine, Xiaoshan Hospital, \\ Xiaoshan, China \\ Contributions: (I) Conception and design: Q Zhang, X Wu; (II) Administrative support: J Yang; (III) Provision of study materials or patients: Q Zhang; \\ (IV) Collection and assembly of data: Q Zhang, J Yang; (V) Data analysis and interpretation: Q Zhang, X Wu; (VI) Manuscript writing: All authors; \\ (VII) Final approval of manuscript: All authors. \\ Correspondence to: Jie Yang. Department of Cardiovascular Medicine, Xiaoshan Hospital, No. 728 Yucai North Road, Xiaoshan, China. \\ Email: zhangqiufeng01@sina.com.
}

Background: MicroRNA (miRNA), which participates in various physiological and pathological processes, is a highly conserved small RNA sequence. This study aimed to investigate the role of miR-194-5p in hypoxia/reoxygenation (H/R)-induced cardiomyocyte apoptosis and myocardial ischemia/reperfusion (I/R) injury.

Methods: We set up an H/R H9c2 cell model in vitro and an I/R mouse model in vivo. Then, cell vitality, apoptosis, and histopathological evaluation were conducted. Reactive oxygen species (ROS) generation and the activity of superoxide dismutase (SOD) and malondialdehyde (MDA) were examined by 2',7'-Dichloro dihydrofluorescein diacetate (H2DCFDA), and enzyme-linked immunosorbent assay (ELISA), respectively. The level of creatine kinase isoenzyme (CK-MB), cardiac troponin I (cTnI), myoglobin $(\mathrm{Mb})$ is examined by ELISA. The expression of Caspase-3, cleaved-Caspase-3, Bax, Bcl-2, phosphatase and tensin homolog deleted on chromosome ten (PTEN), and protein kinase B (AKT) was analyzed by western blot.

Results: Data showed the expression of miR-194-5p was decreased in H/R-induced H9c2 cells and I/ R-induced mouse. Conversely, overexpression of miR-194-5p could improve cardiomyocyte damage in ischemic models in vivo and in vitro. Furthermore, mitogen-activated protein kinase 1 (MAPK1) was found as a direct target of miR-194-5p, which negatively regulated the expression of MAPK1. The up-regulation of MAPK1 inhibited the myocardial protection previously observed by miR-194-5p.

Conclusions: Our study shows overexpression of miR-194-5p protects against H/R injury in vitro and cardiac I/R injury in vivo, which involves the inhibition of cardiac apoptosis and oxidative stress by targeting MAPK1 expression via PTEN/AKT pathway. These findings supply novel insights into potential therapeutic targets for cardiovascular diseases.

Keywords: MicroRNA; apoptosis; myocardial injury; oxidative stress

Submitted Sep 06, 2020. Accepted for publication Apr 17, 2021.

doi: 10.21037/atm-21-807

View this article at: http://dx.doi.org/10.21037/atm-21-807

\section{Introduction}

Cardiovascular disease is a significant threat to human health and the second leading cause of death after the World Health Organization (WHO, 2017). Acute myocardial infarction is the leading cause of cardiovascular death worldwide (1), which results in cardiomyocyte damage and cardiac dysfunction. Early and exact diagnosis and appropriate therapeutic intervention may reduce cardiac mortality (2). Myocardial reperfusion is the most effective treatment intervention for ischemic heart disease (3), of which the main treatments are thrombus and percutaneous coronary intervention (4). However, reflux of blood will be 
accompanied by myocardial I/R damage, causing apoptosis or necrosis of cardiomyocytes, and cardiac arrest (5). Therefore, effective methods to prevent and mitigate I/R injury have become the main topic in myocardial protection research.

MicroRNAs (miRNAs) are a short, highly conserved non-coding RNA fragment that can effectively disturb protein translation by negatively regulating gene expression through mRNA splicing (6). A wealth of evidence suggests miRNA are potential and viable candidates of clinical biomarkers for the detection and screening of human diseases $(7,8)$. miRNA can endure repeated freeze-thaw cycles, hemodialysis, and even nuclease digestion (9), which play an essential role in the pathophysiological process of cardiovascular disease and myocardial I/R injury (10). Although miR-194-5p has been shown to have anti-inflammatory $(11,12)$ and anti-tumor effects $(13)$, its regulatory mechanism in cardiovascular disease is still unclear.

In the present study, we developed a cardiomyocyte $\mathrm{H} / \mathrm{R}$ model and a mouse I/R model to evaluate early ideal biomarkers in AMI patients. We sought to explore the potential therapeutic role of miR-194-5p in myocardial injury. We present the following article in accordance with the ARRIVE reporting checklist (available at http://dx.doi. org/10.21037/atm-21-807).

\section{Methods}

\section{Cell culture}

H9c2 cells were obtained from China Center for Type Culture Collection (CCTCC, Wuhan, China), and cultured in DMEM, high glucose, medium (Gibco, CA, USA) supplemented with 10\% FBS (Gibco) and 1\% PenicillinStreptomycin liquid (Gibco) in a $5 \% \mathrm{CO}_{2}$ incubator (Thermo, Waltham, USA) at $37^{\circ} \mathrm{C}$.

\section{Hypoxia/reoxygenation (H/R) model}

H9c2 cells were cultured in DMEM medium (Gibco) supplemented with $10 \%$ FBS (Gibco) under anaerobic conditions ( $95 \%$ air, $\left.5 \% \mathrm{CO}_{2}, 37{ }^{\circ} \mathrm{C}\right)$. Post 48 hours. The cells were exposed to $95 \% \mathrm{~N}_{2}, 5 \% \mathrm{CO}_{2}$, and $1 \% \mathrm{O}_{2}$ serum-free DMEM without glucose and sodium acetate at $37^{\circ} \mathrm{C}$ for 24 hours. Subsequently, cells were reoxygenated to mimic ischemia-induced cardiomyocyte injury by incubating $37{ }^{\circ} \mathrm{C}$ with DMEM containing $10 \%$ in $95 \%$ air and $5 \% \mathrm{CO}_{2}$ for 12 hours.

\section{In vitro transfection}

H9c2 cells were seeded in a 6 -well plate. Following the manufacturer's instructions, miR-194-5p-mimic/ NC (Sangon, Shanghai, China), or pcDNA-MAPK1 (GenePharma, Shanghai, China) was transfected into these cells using Lipofectamine ${ }^{\mathrm{TM}} 2000$ Transfection Reagent (Invitrogen, Carlsbad, USA), followed by incubation with $5 \% \mathrm{CO}_{2}$ at $37^{\circ} \mathrm{C}$ for 6 hours. Finally, the transfected cells are used to $\mathrm{H} / \mathrm{R}$ exposure and evaluate their transfection efficiency.

\section{Cell viability}

H9c2 cells $(20 \mu \mathrm{L})$ were inoculated on a 96-well plate at a density of $1 \times 10^{3}$ cells/well. After three days, $20 \mu \mathrm{L}$ of MTT solution $(5 \mathrm{mg} / \mathrm{mL}, \mathrm{PH} 7.4)$ was added to each well and incubated for four hours at $37^{\circ} \mathrm{C}$. Then, the supernatant was removed, and $150 \mu \mathrm{L}$ DMSO was added to dissolve the crystals fully. The absorbance at $490 \mathrm{~nm}$ was measured using a Multiskan Sky microplate reader (Thermo, Waltham, USA).

\section{Flow cytometry}

The percentage of early apoptosis rate was decided using the Apoptosis Detection Kit (Vazyme, Nanjing, China). Succinctly, the designed H9c2 cells were plated in 24-well plates for 48 hours. Then, the cells were harvested and costained with $5 \mu \mathrm{L}$ FITC and $1 \mu \mathrm{L}$ PI for 15 minutes at room temperature in the dark. A flow cytometer measures the cellular fluorescence (Thermo).

\section{Quantitative real-time PCR (qRT-PCR)}

Total RNA was extracted from cardiac tissues or myocardial cells using TRIzol reagent (Invitrogen). The primer sequences were designed from Sangon and listed as below: miR-194-5p, F 5'-CTAGTAC CTAGAGGAACCTTTGAAGACTGTTACAG CTCAGCA-3'，R 5'-AGCTTGCTGAGCTGTA ACAGTCTTCAAAGGTTCCTCTAGGTA-3 '; MAPK1: F 5'-TTTCCTCTGGATCAGCGTGT-3', R 5 '- TGAGATGTCGGGGCT TCT T T-3 '; GAPDH: F 5'-ACCACAGTCCATGCCATCAC-3', R 5'-TCCACCACCCTGTTGCTGT-3'; U6: 
F 5'-ACCCTGAGAAATACCCTCACAT-3', R 5'-GACGACTGAGCCCCTGATG-3'. RNA was reversed into cDNA by a cDNA Synthesis Kit (TaKaRa, Tokyo, Japan). Quantitative amplification was performed using the QuantStudio $^{\mathrm{TM}} 6$ Flex system (Thermo). The $2^{-\Delta \Delta \mathrm{CT}}$ mean (14) was conducted to calculate the relative quantification. GAPDH and U6 as internal control.

\section{Ischemia/reperfusion (I/R) model}

Healthy male c57BL6/J mice ( 8 weeks old, 18-22 g) were obtained from the Animal Experiment Center of Sichuan University (Chengdu, China). Animal protocols in this study were approved by the Animal Care and Use Committee of First People's Hospital of Shangqiu City. They are placed in an environment of $25^{\circ} \mathrm{C}$ and $60 \%$ humidity with a 12-hour light/dark cycle. All steps were approved by and conducted following the Guide for the Care and Use of Laboratory Animals (15). An I/R mouse model (16) was set up by ligating the left anterior descending coronary artery (LAD). The mice were anesthetized with $2 \%$ isoflurane (RWD, Kent, USA) and fixed to the operating table. We opened the left thoracic cavity to expose the heart and ligate 30 minutes, around the LAD with a 6-0 silk suture, then reperfusion 180 minutes. Do the same procedure without LAD for the sham group. Ligation tissue and serum samples were collected 24 hours after induction I/R, the tissue, and serum samples were collected for in vivo study.

\section{In vivo transfection}

miR-194-5p adeno-associated virus (AAV) vectors were constructed by OBiO (Shanghai, China), of which reference sequence as below: 5'-UGUAACAGCAACUCCAUGUGGA-3'. AAV scrambled control (AAV-SC) as a control, of which sequence as below: 5'-UUCUCCGAACGUGUCACGUTT-3'. 2 weeks before I/R induction, miR-194-5p AAV and AAV$\mathrm{SC}$ were intravenously injected into the tail vein (17). Mice were divided into four groups $(\mathrm{n}=8)$ : Sham, I/R, I/R + AAV$\mathrm{SC}$, and $\mathrm{I} / \mathrm{R}+\mathrm{AAV}$.

\section{Enzyme-linked immunosorbent assay (ELISA) assay}

Cell supernatants and serum samples were centrifuged and collected, the quantification of SOD and MDA were detected using commercial ELISA kits (Thermo), according to the manufacturer's instructions.

\section{Reactive oxygen species (ROS) generation}

The ROS production was measured as described before (18). H9c2 cells were seeded into a 12-well plate and then treated with $5 \mathrm{mmol} / \mathrm{L} \mathrm{N}$-acetyl-L-cysteine (NAC, Invitrogen) for 1 hour. Then, the medium was replaced with serumfree medium, and $10 \mu \mathrm{M}$ DCFH-DA (Sigma) was added to incubate cells in the dark for 20 minutes at $37^{\circ} \mathrm{C}$. Finally, the treated cells were washed with PBS, a flow cytometer that immediately assessed DCF fluorescence (Thermo) and analyzed by FlowJo ${ }^{\mathrm{TM}}$ software (Becton, Dickinson \& Company, New Jersey, USA).

\section{Hematoxylin and eosin (HE) staining}

The mouse's heart tissue was fixed in $10 \%$ buffered formalin and embedded in paraffin. The 4- $\mu \mathrm{m}$ sections were then stained with a HE solution for the pathological analysis. Confocal microscopy (Leica, Wetzlar, Germany) was used to examine the changes in the tissue structure of the complex.

\section{TdT-mediated dUTP nick end labeling (TUNEL) staining}

After the paraffin sections were deparaffinized and hydrated, which were treated 30 minutes with DNase proteinase $\mathrm{K}$ (Thermo), the sections were stained and were performed with FragEL ${ }^{\mathrm{TM}}$ DNA Fragmentation Detection Kit (Millipore, Massachusetts, USA). Five visible areas were randomly selected under the Leica microscope, and the number of apoptotic cells was counted.

\section{Western blot}

Total protein was lysed with radioimmunoprecipitation (RIPA) buffer (Beyotime, Haimen, China). According to the manufacturer's instructions, a BCA protein assay kit quantified the protein concentrations (Abcam, Cambridge, UK). Then about $50 \mu \mathrm{g}$ proteins separate using $12 \%$ SDS PAGE gels and transferred onto polyvinylidene fluoride (PVDF) membranes. Post blocking with 5\% non-fat milk for 30 minutes at $4{ }^{\circ} \mathrm{C}$, the membranes were incubated with primary antibodies as below: MAPK1 antibody (\#9108, CST), Bcl-2 antibody (ab59348, Abcam), Bax antibody (ab32503, Abcam), Cleaved-Caspase-3 antibody (ab184784, Abcam), PENT antibody (\#9188, CST), p-AKT antibody (\#4060, CST), and AKT (\#4691, CST). The next day, the membranes were incubated with the matching secondary antibody one hour at room temperature. Finally, the bands 
A

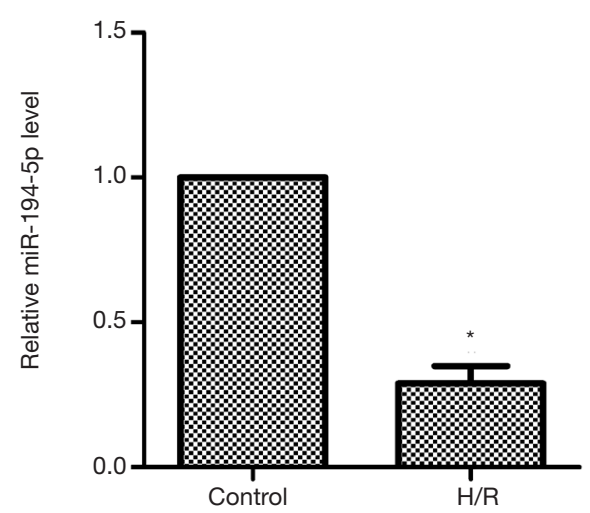

C
B
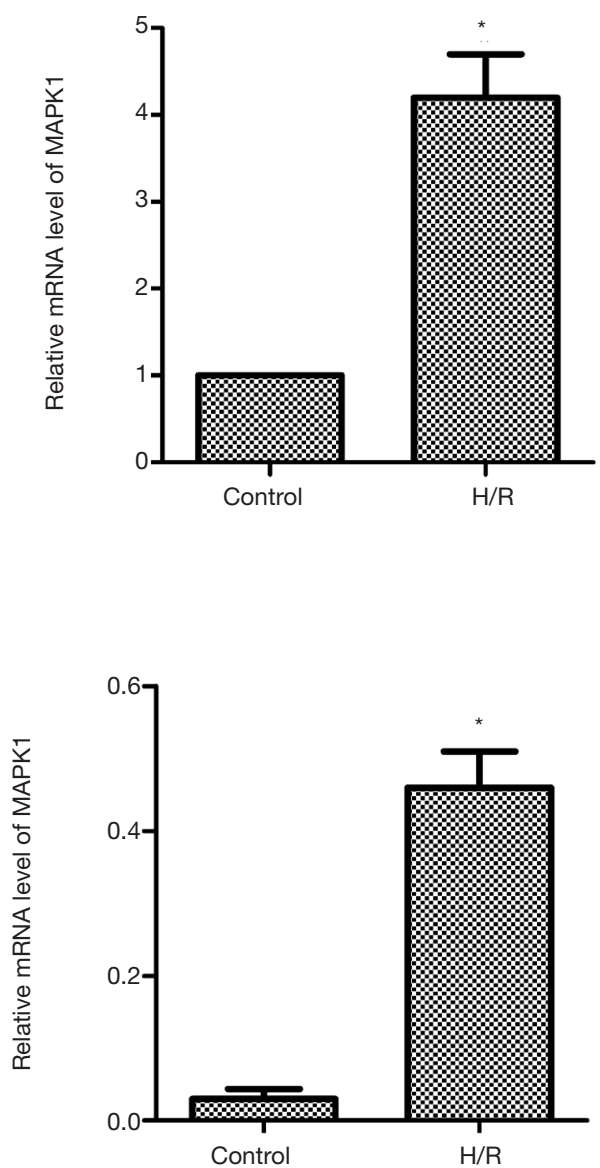

Figure 1 miR-194-5p low-expression and MAPK1 high-expression in H9c2 cells. The mRNA level of miR-194-5p (A) or MAPK1 (B) in normal and H/R-induced H9c2 cells were detected by qRT-PCR. (C) The protein level of MAPK1 was detected by western blot. Data are depicted as mean \pm SEM. * $\mathrm{P}<0.05$ vs. control. All procedures are performed greater than or equal to 3 times.

were exposed with enhanced ECL reagents (Sangon). $\beta$-actin as the internal control.

\section{Statistical analysis}

$\mathrm{P}<0.05$ was considered a statistically significant difference. SPSS performed statistical analysis 22.0 (IBM, Armonk, USA), and data were presented as the mean \pm SEM. The student's $t$-test analyzed comparison difference or one-way ANOVA, followed by Tukey's post hoc test.

\section{Results}

\section{Down-regulation of miR-194-5p and up-regulation of MAPK1 in H/R-induced H9c2 cells}

Post H/R induced h9c2 cells; the mRNA expression of miR- 194-5p was markedly lower than the control group (Figure 1A). Then we detected the MAPK1 expression using qPCR and western blot, respectively. As shown in Figure 1B, the mRNA level of MAPK1 was markedly higher. Similarly, the protein level of MAPK1 was increased compared with the control group (Figure 1C).

\section{miR-194-5p directly targeted MAPK1 in H/R-induced} H9c2 cells

TargetScan tool showed the target sites between miR194-5p and MAPK1 (Figure 2A). As shown in Figure 2B, the luciferase activity of wt 3'-UTR-MAPK1 reduced, but mut 3'-UTR-MAPK1 luciferase activity hardly changed. Furthermore, miR-194-5p mimic was transfected into H/ R-induced H9c2 cells for 24 hours, and the expression of 
A

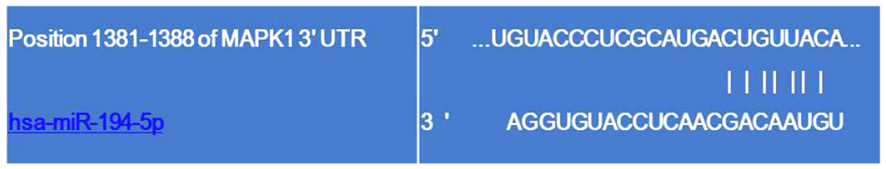

B

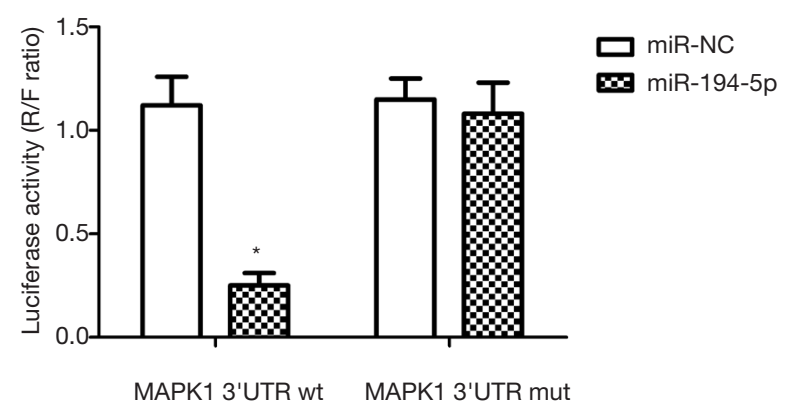

D

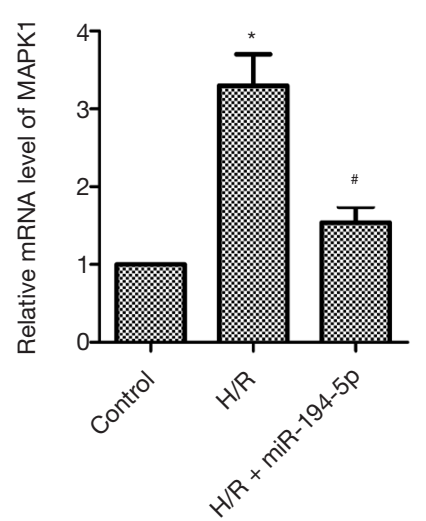

$\mathrm{E}$

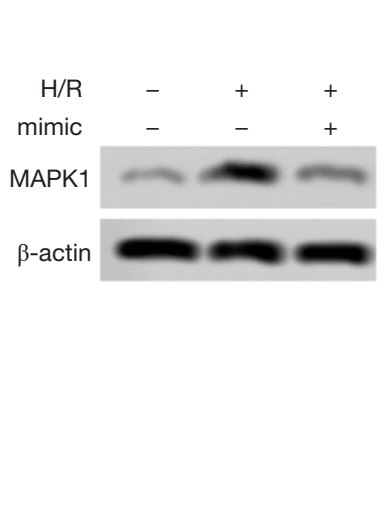

C
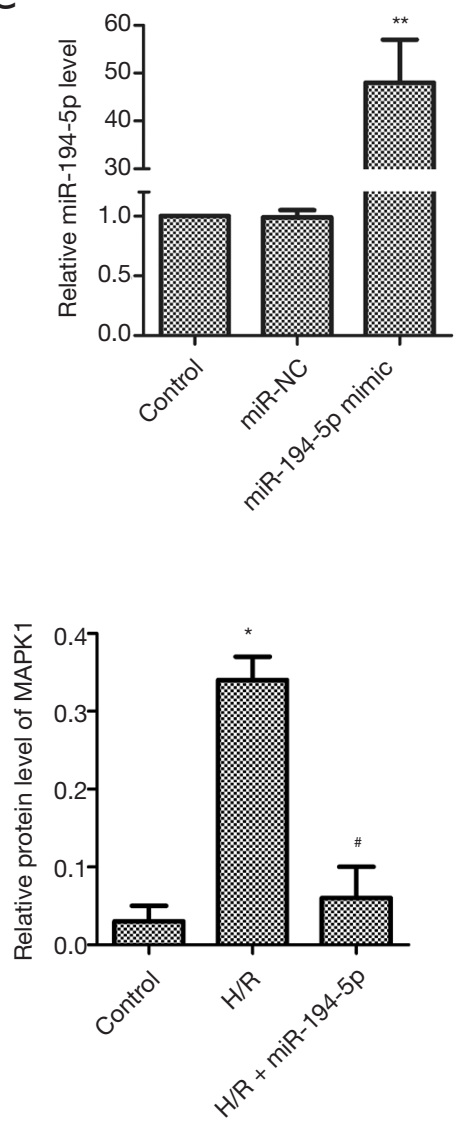

Figure 2 miR-194-5p directly targets MAPK1. (A) Prediction of the potential binding site of miR-194-5p on MAPK1-wt. (B) Relative luciferase activity of MAPK1-wt/mut was assessed in H9c2 cells transfected with miR-194-5p mimic/NC by a dual-luciferase reporter. *, $\mathrm{P}<0.05$ vs. miR-194-5p NC. (C) H9c2 cells were transfected with miR-194-5p mimic or NC 24 hours later, the mRNA level of miR-194$5 \mathrm{p}$ was detected by qRT-PCR. **, $\mathrm{P}<0.01$ vs. control. (D,E) The protein expression of MAPK1 is examined by qRT-PCR and western blot, respectively. ${ }^{*}, \mathrm{P}<0.05$ vs. control; ${ }^{*}, \mathrm{P}<0.05$ vs. H/R. Data are depicted as mean $\pm \mathrm{SEM}$; all procedures are performed greater than or equal to 3 times.

miR-194-5p was elevated (Figure 2C), the mRNA level of MAPK1 was significantly decreased (Figure 2D), which was consistent with the protein changes of MAPK1 (Figure 2E).

\section{High expression of miR-194-5p promotes cell viability and inhibits apoptosis in H/R-induced $H 9 c 2$ cells}

Compared with the control, H9c2 cells exposed to H/R markedly reduced cell viability, and overexpression of MAPK1 further reduced cell viability. Delightful, elevated miR-194-
$5 \mathrm{p}$ significantly increased the cell viability of $\mathrm{H} 9 \mathrm{c} 2$ cells (Figure $3 A$ ). We then used flow cytometry to examine the effect of miR-194-5p transfection on H/R-induced H9c2 cell apoptosis. As shown in Figure 3B,C, the apoptotic rate of $\mathrm{H} 9 \mathrm{c} 2$ cells conspicuously increased after H/R or H/R+MAPK1, compared with control. However, the miR-194-5p mimic decreased cardiomyocyte apoptosis. Moreover, the labeled protein of apoptosis (Caspase-3, Bax, and Bcl-2) was detected by western blot, and the expression of cleaved-caspase- 3 and the value of $\mathrm{Bax} / \mathrm{Bcl}-2$ was significantly decreased (Figure 3D). 
A

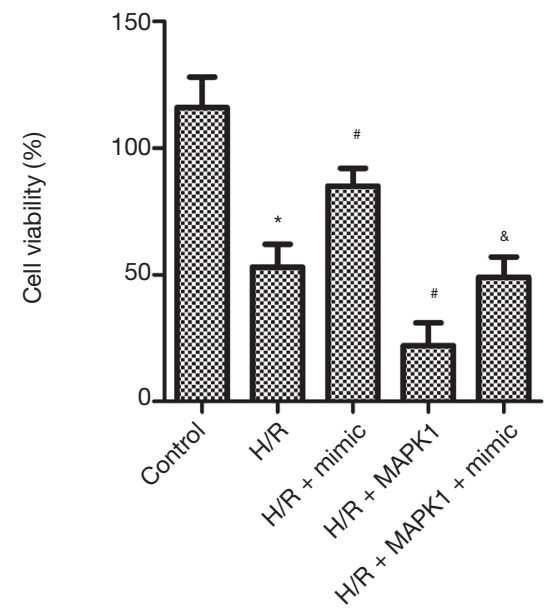

B

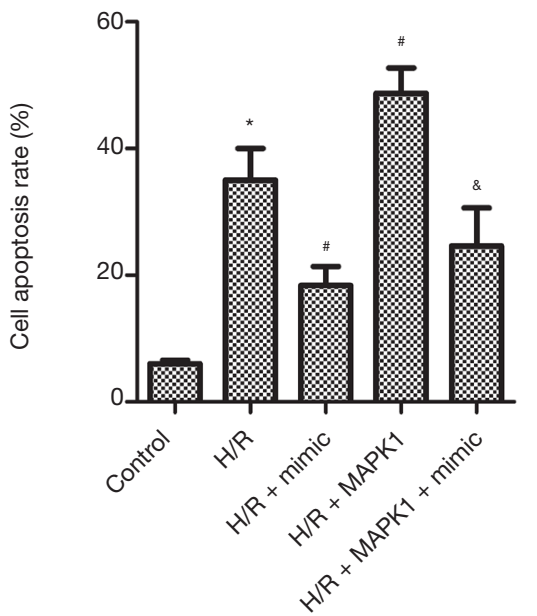

C

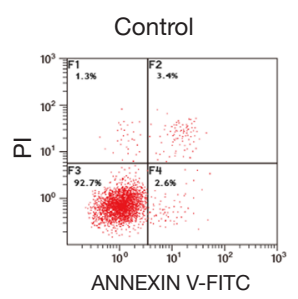

D

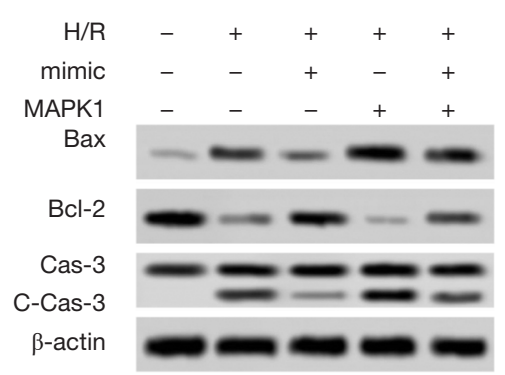

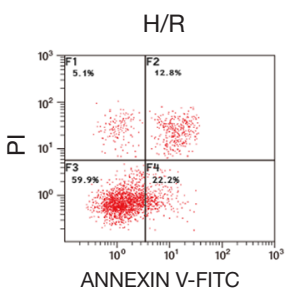

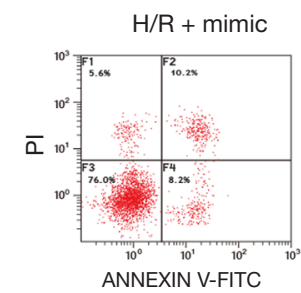

ANNEXIN V-FITC
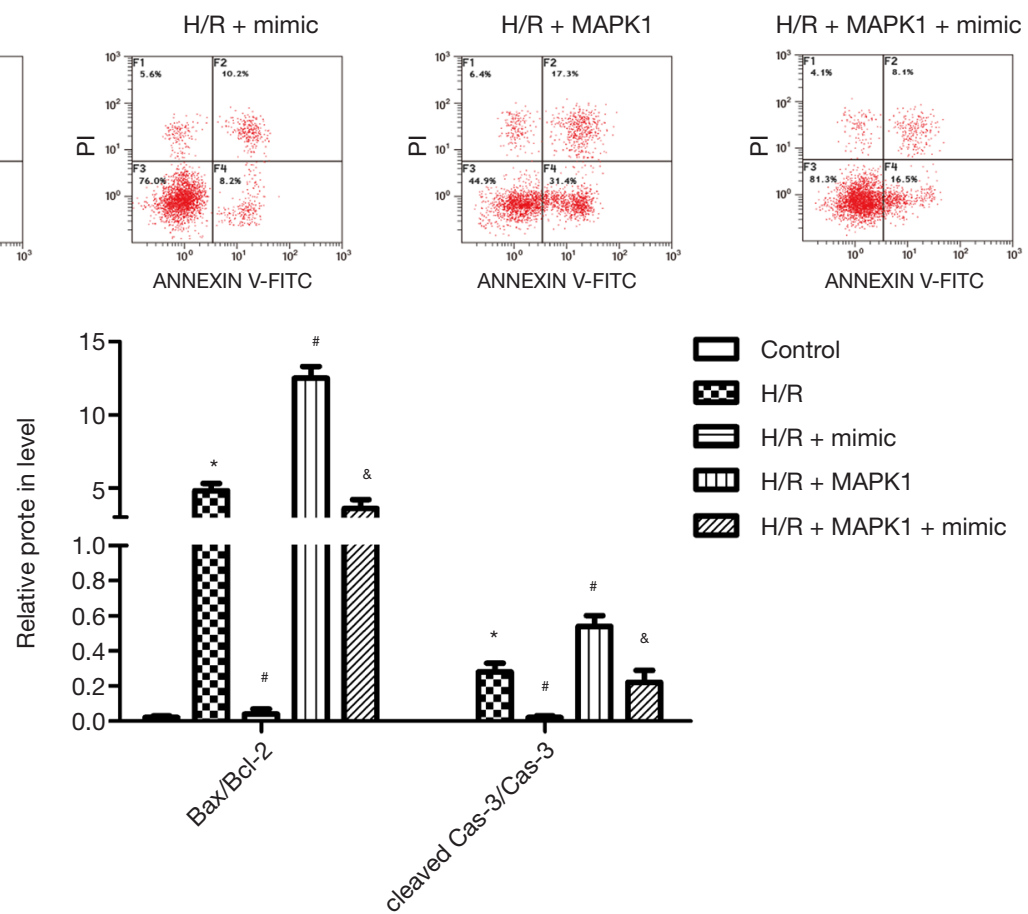

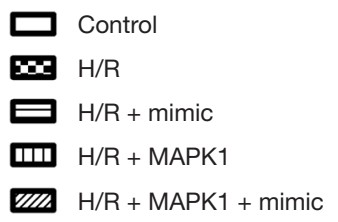

$\mathrm{H} / \mathrm{R}+\mathrm{MAPK} 1+$ mimic

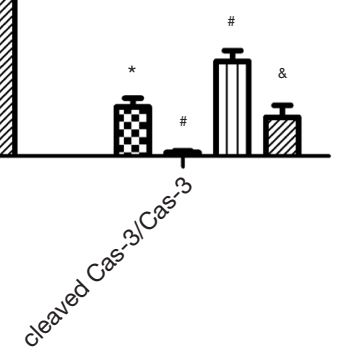

Figure 3 Effect of miR-194-5p on cell viability and apoptosis of H9c2 cells. (A) The cell viability of H9c2 cells was measured used MTT; $(\mathrm{B}, \mathrm{C})$ the apoptotic rate of $\mathrm{H} 9 \mathrm{c} 2$ cells is examined by flow cytometry; (D) the protein expression of Bax, Bcl-2, and cleaved-Caspase-3 was examined by western blot. Data are depicted as mean \pm SEM, *, $\mathrm{P}<0.05$ vs. control; ${ }^{*}, \mathrm{P}<0.05$ vs. H/R; ${ }^{\circledR}, \mathrm{P}<0.05$ vs. H/R + MAPK1. All procedures are performed greater than or equal to 3 times.

\section{High expression of miR-194-5p blocked H/R-induced oxidative stress}

As shown in Figure 4A,B, the levels of SOD and MDA were also examined by ELISA. The result showed that SOD activity decreased, and the activity of MDA increased post
H9c2 cells exposed to H/R or MAPK1 overexpression, compared with control. Also, H/R exposes stimulated ROS generation (Figure 4C). However, the activity change of these markers was reversed by miR-194-5p transfection (Figure 4A,B,C,D). 
A

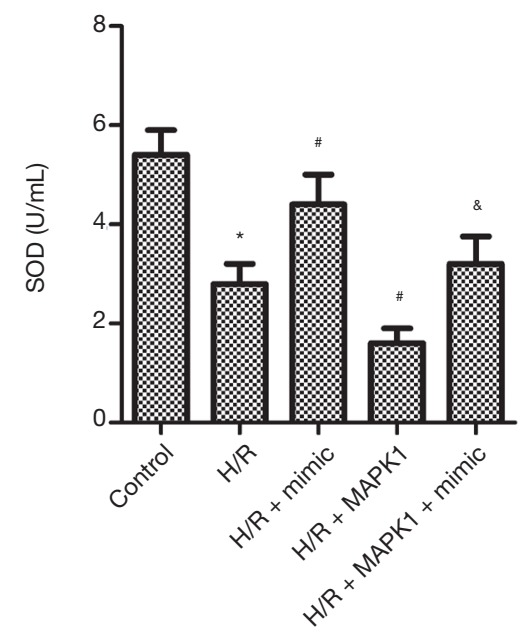

B

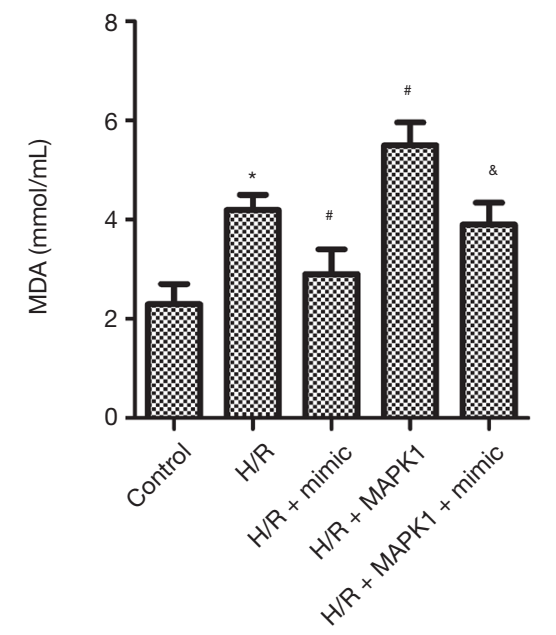

C

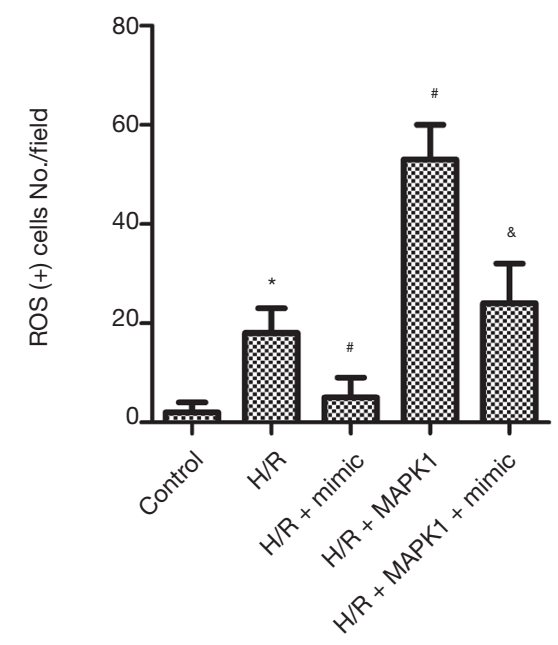

D

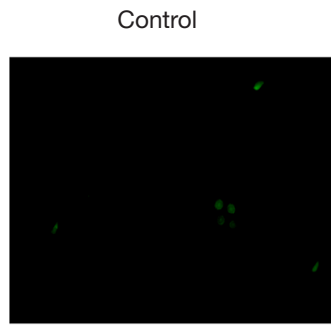

$\mathrm{H} / \mathrm{R}$

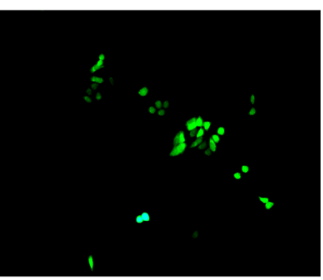

$H / R+$ mimic

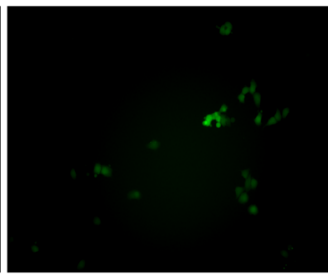

$H / R+M A P K 1$

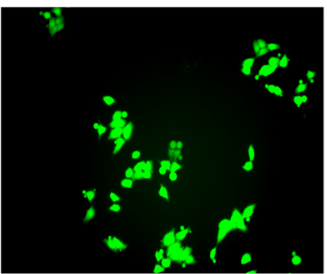

$\mathrm{H} / \mathrm{R}+\mathrm{MAPK} 1+$ mimic

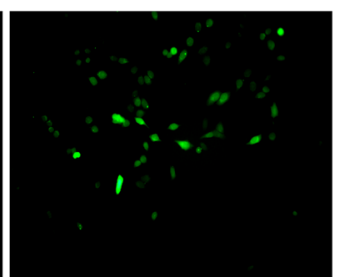

Figure 4 Effect of miR-194-5p on H/R-induced oxidative stress. (A,B) The activities of SOD and MDA were measured by ELISA assay; (C,D) H2DCFDA examined ROS production; images were magnified at $\times 200$. Data are depicted as mean $\pm \mathrm{SEM}$. *, $\mathrm{P}<0.05$ vs. control; ${ }^{*}, \mathrm{P}<0.05$ vs. $\mathrm{H} / \mathrm{R} ;{ }^{\&}, \mathrm{P}<0.05$ vs. H/R + MAPK1. All procedures are performed greater than or equal to 3 times.

High expression of miR-194-5p attenuates H/R injury via MAPK1/PTEN/Akt signaling patbway in $\mathrm{H} 9 \mathrm{c} 2$ cells

To explore the relationship between miR-194-5p and signaling pathway in H/R-treated H9c2 cells, the expression of MAPK1, PTEN, and p-AKT were evaluated by western blot. Western blot results (Figure $5 A, B$ ) showed that H/R treatment increased MAPK1 protein expression, which was reversed by miR-194-5p mimic. Next, the effects of miR194-5p on PTEN/AKT pathway were further investigated. Compared with H/R group, miR-194-5p mimic obviously decreased PTEN expression and increased p-AKT/AKT ratio (Figure $5 A, C, D$ ).

\section{Down-regulation of miR-194-5p and up-regulation of MAPK1 in I/R-induced myocardial tissues}

Compared with the control group, the mRNA expression of miR-194-5p was markedly lower (Figure 6A). Similarly, we also detected the MAPK1 expression at the mRNA and protein levels, respectively. As shown in Figure $6 B, C$, the mRNA level of MAPK1 was markedly higher, and MAPK1 protein was increased, compared with the control group.

\section{High expression of miR-194-5p alleviated I/R injury in mice}

To further confirm the myocardial protective role of miR-194-5p in vivo, we established a mouse model of I/ $\mathrm{R}$ injury after miR-194-5p-AAV injection. As shown in Figure $6 D, E, F$, the expression of cTnI, CK-MB, and $\mathrm{Mb}$ were conspicuously increased following I/R. However, miR-194-5p overexpression significantly decreases the serum level of these markers. As shown in Figure $7 \mathrm{~A}$, compared with the complete morphological structure of the control group, I/R caused myocardial fiber breakage, cellular edema, and neutrophil infiltration. As expected, high expression of miR-194-5p repaired 
A
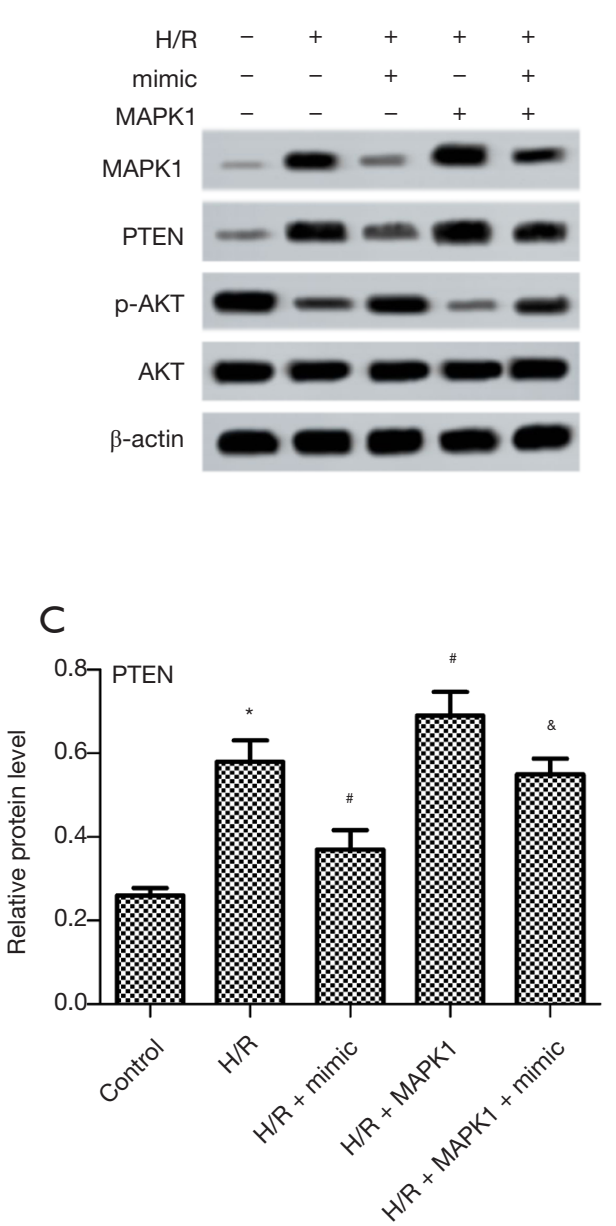

B
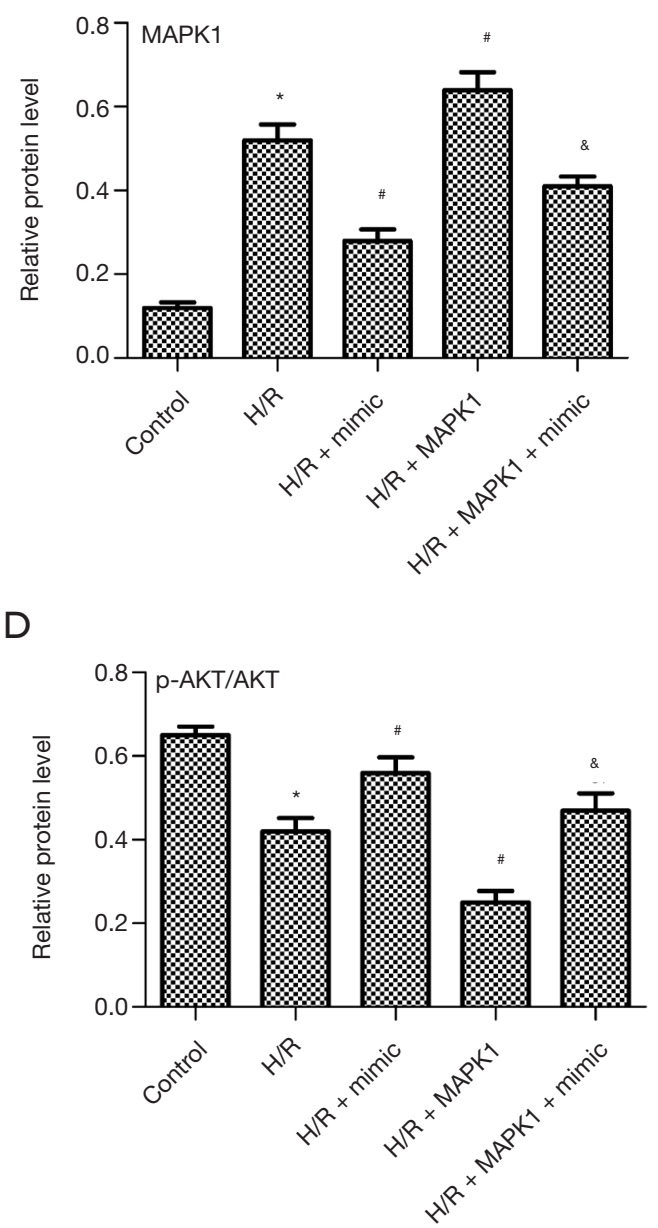

Figure 5 Effect of miR-194-5p on MAPK1/PTEN/AKT pathway in H/R-induced H9c2 cells. (A,B,C,D) The protein expression of MAPK1, PTEN, p-AKT, and AKT were examined by western blot. Data are depicted as mean \pm SEM. *, $\mathrm{P}<0.05$ vs. control; *, $\mathrm{P}<0.05$ vs. H/ $\mathrm{R} ;{ }^{8}, \mathrm{P}<0.05 v s . \mathrm{H} / \mathrm{R}+$ MAPK1. All procedures are performed greater than or equal to 3 times.

myocardial tissue injury. As shown in Figure $7 B$, we used the TUNEL method to detect the apoptotic rate of myocardial tissue. Compared with the control group, the number of apoptotic cells increased significantly after I/ R. Conversely, elevated miR-194-5p reduced the number of apoptotic cells in myocardial tissue. Still, the protein expression of Caspase-3, Bax, and Bcl-2 was detected by western blot. The result showed that the cleaved-Caspase- 3 expression and the value of $\mathrm{Bax} / \mathrm{Bcl}-2$ was significantly decreased after miR-194-5p AVV transfection, compared with I/R (Figure 7C,D). Additionally, Compared with control, SOD decreased (Figure $7 E$ ), and MDA (Figure $7 F$ ) increased following I/R, but miR-194-5p AVV reversed these changes. Also, compared with $\mathrm{I} / \mathrm{R}$, we also found that
miR-194-5p AVV decreased the PTEN expression and increased p-AKT/AKT ratio (Figure $7 G$ ). These results, together, indicate that miR-194-5p plays an important role in cardiomyocyte injury in I/R mmice.

\section{Discussion}

At present, elucidating the molecular mechanism of cardiovascular disease is an urgent clinical challenge. To the best of our knowledge, this is the first time to provide evidence that abnormal expression of miR-194-5p may be mediated by myocardial ischemic injury, and overexpression of miR-194-5p protects against cardiac injury in vivo and in vitro. This study suggests miR-194-5p confers cardiac 
A

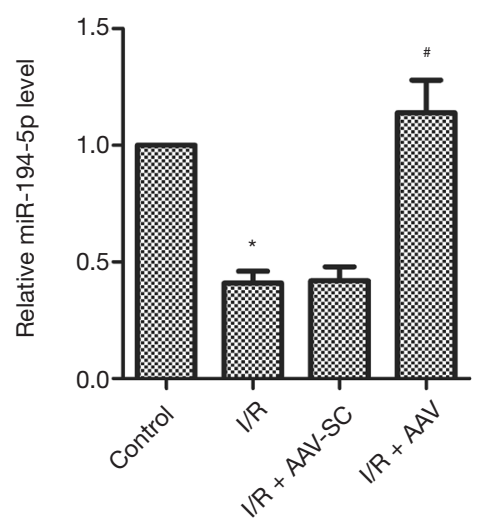

D

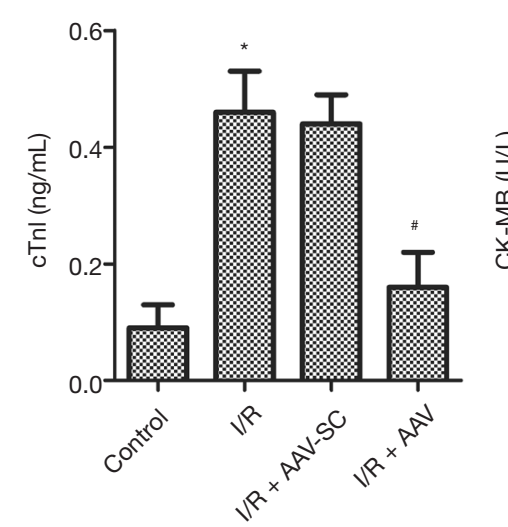

B

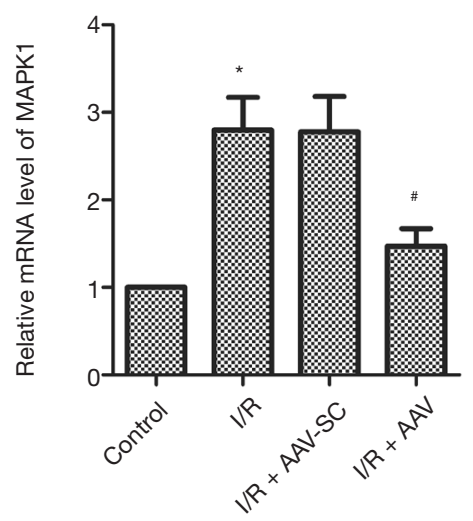

$E$

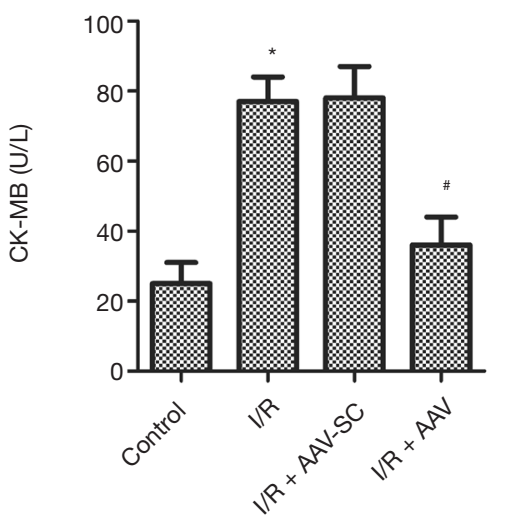

C
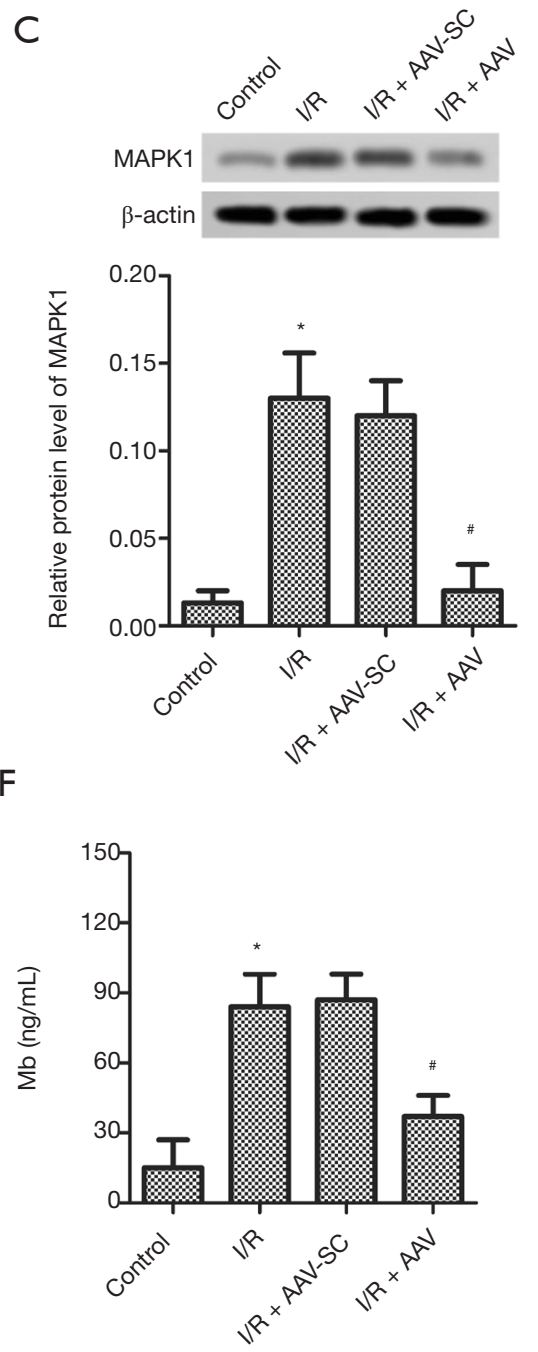

Figure 6 Effect of miR-194-5p on the myocardial injury. (A) The mRNA level of miR-194-5p in myocardial tissues. The mRNA (B) and protein level (C) of MAPK1 in myocardial tissues. Myocardial injury markers of cTnI (D), CK-MB (E), and Mb (F) were examined by ELISA assay. Data are depicted as mean $\pm \mathrm{SEM}, \mathrm{n}=8$. *, $\mathrm{P}<0.05$ vs. control; ${ }^{\#}, \mathrm{P}<0.05$ vs. I/R. All procedures are performed greater than or equal to 3 times.

protection by targeting MAPK1.

Ischemic heart disease is a common disease in the clinical cardiovascular system (19). Various methods of revascularization can effectively improve myocardial damage or necrosis caused by ischemia (3). Unfortunately, so far, there is no effective clinical strategy to avoid the risk of reperfusion injury, limiting the prognosis of coronary heart disease treatment. Furthermore, miRNA expression changes abnormally in pathological stimulation or tissue injury (20). Increasingly evidence shows miRNA participates in the regulatory process of ischemic injury $(10,21)$. Nevertheless, the biological role of miR-194-5p in cardiomyocytes is still poorly understood. In this study, we found miR-194-5p expression was decreased both in $\mathrm{H} 9 \mathrm{c} 2$ cells exposed to $\mathrm{H} / \mathrm{R}$ and in cardiomyocytes of a mouse I/R model. In addition, our study also showed that up regulation of miR-194-5p could protect cardiomyocytes from apoptosis and oxidative stress in vivo and in vitro.

Apoptosis is an essential physiological feature of early I/ $\mathrm{R}$ (22), which triggers more severe myocardial injury and heart failure (23). Apoptosis is a process of programmed cell death; Bcl-2 family members mediate apoptosis by controlling the mitochondrial membrane (24). We found miR-194-5p up-regulation could protect cardiomyocytes 
A

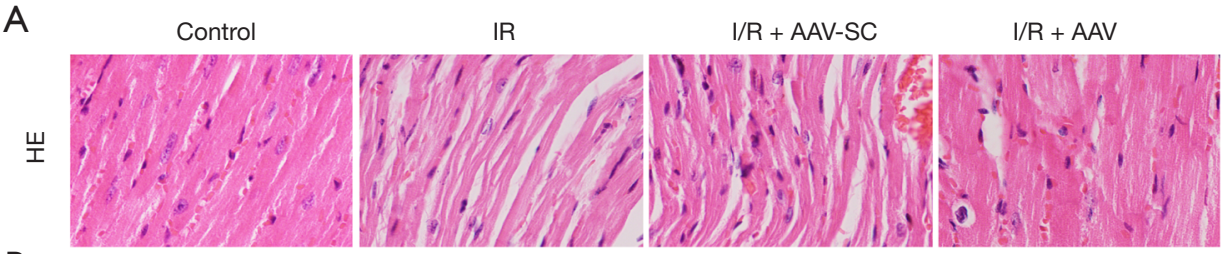

B

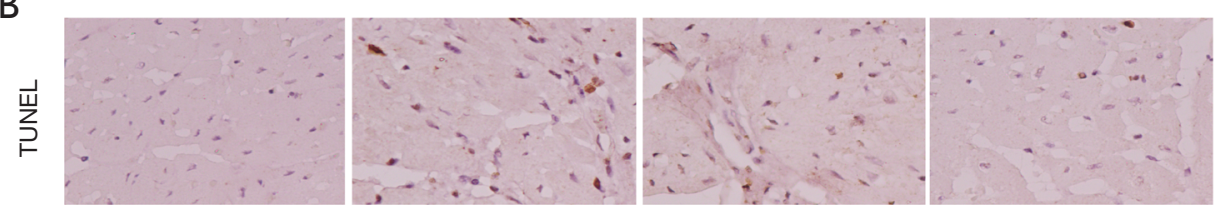

D

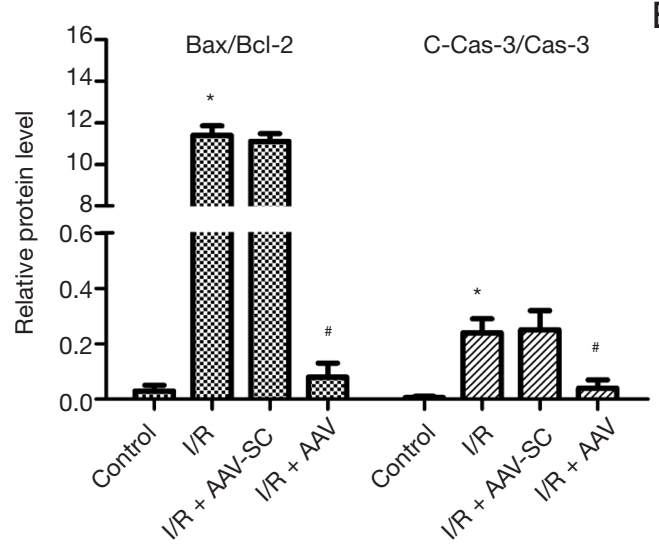

E

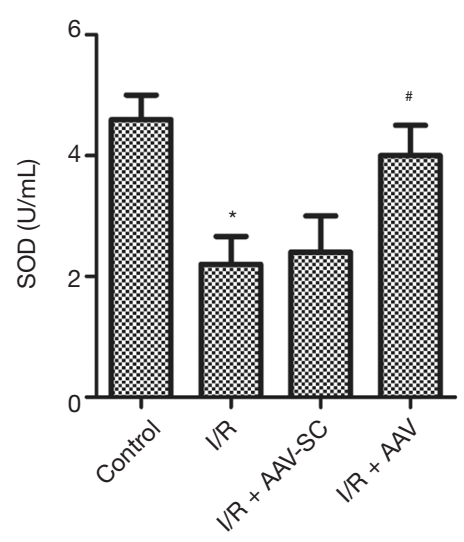

C

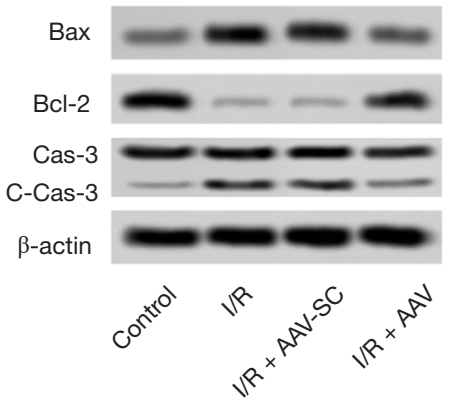

$\mathrm{F}$

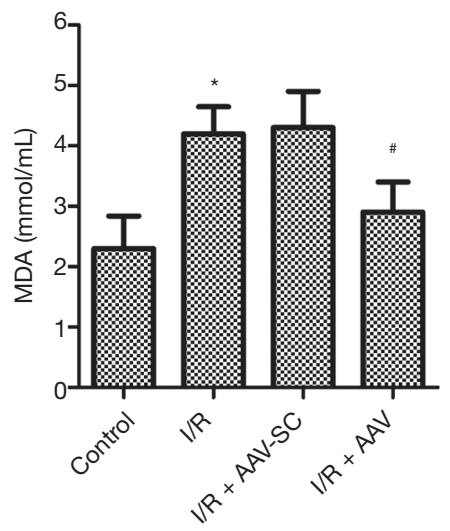

G
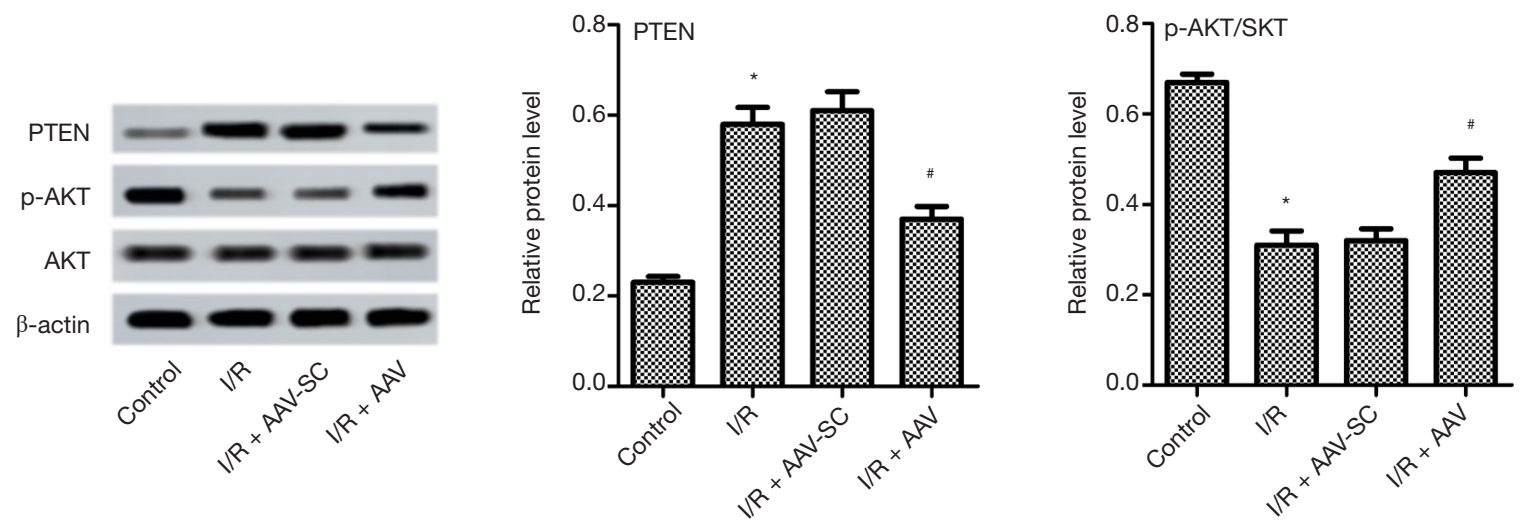

Figure 7 Effect of miR-194-5p on I/R injury in mice. (A) Myocardial tissues were stained using HE; images were magnified at $\times 400$; (B) the apoptosis of myocardial tissues was detected using TUNEL staining, images were magnified at $\times 400$; (C,D) the protein expression of Bax, Bcl-2, and cleaved-Caspase-3 was examined by western blot; the activities of SOD (E) and MDA (F) were measured by ELISA assay; (G) the protein expression of PTEN, p-AKT, and AKT were examined by western blot. Data are depicted as mean \pm SEM. n=8. *, P $<0.05$ vs. control; ", $\mathrm{P}<0.05$ vs. I/R. All procedures are performed greater than or equal to 3 times.

from apoptosis in vivo and in vitro through flow cytometry and TUNEL staining, the expression of cleaved-caspase-3, and the value of Bax/Bcl-2 was significantly decreased. During I/R injury, excessive ROS generation accompanies reperfusion, which leads to further myocardial injury (25).
In our study, we evaluated the level of oxidative stress, including increased MDA and decreased SOD after reperfusion (26). Up-regulation of miR-194-5p led to the decrease of SOD activity, the increase of MDA activity, and the inhibition of ROS production. These results further 
confirmed the cardiac protective effect of miR-194-5p in vitro and in vivo.

Mitogen-activated protein kinases (MAPKs) are a family of serine/threonine protein kinases, which mediate fundamental biological processes and cellular responses to external stress signals, including proliferation (27), apoptosis (28), or inflammation (29). Specifically, Our study proved that MAPK1 is a functional target gene of miR-194$5 \mathrm{p}$. Nevertheless, since the mediating effects of miRNA on gene expression are specific, it is unclear whether MAPK1 continues to act as a target gene of miR-194-5p during H/R or I/R processes. Our results clearly showed miR194-5p regulated MAPK1 expression in cardiomyocytes, explaining the effects of miR-194-5p mediated cardiac protection. PTEN is a tumor suppressor that has been extensively studied (30). The MAPK pathway (31) and PI3K/AKT pathway are its downstream signaling pathways (32). After dephosphorylation of most PTEN, the cell cycle is blocked in the G1 phase through the PI3K/AKT pathway (32). According to reports, the PI3K/ AKT pathway is involved in a variety of cardiovascular diseases, regulating survival, proliferation, apoptosis, and cardiomyocyte hypertrophy and contraction (33). On the other hand, the activation of PTEN/AKT signal can reduce oxidative stress damage and reduce cardiomyocyte apoptosis in myocardial injury $(34,35)$. Our results showed that miR194-5p inhibits MAPK1 and PTEN expression, enhances AKT phosphorylation in H/R or I/R. Additionally, MAPK1 overexpression reversed the effect of miR-194-5p on PTEN and AKT expression, indicating miR-194-5p protects against cardiomyocyte injury through MAPK1/PTEN/ AKT pathway in vivo and in vitro.

\section{Conclusions}

Hence, $\mathrm{miR}-194-5 \mathrm{p}$ played a pivotal role in cardiac injury; our study supplied a new biochemical index for the treatment of cardiovascular disease. Although we found that miR-194-5p overexpression could inhibit the phosphorylation activity of $\mathrm{p} 65$ in vivo, its potential molecular mechanism still needs further research.

\section{Acknowledgments}

Funding: None.

\section{Footnote}

Reporting Checklist: The authors have completed the ARRIVE reporting checklist. Available at http://dx.doi. org/10.21037/atm-21-807

Data Sharing Statement: Available at http://dx.doi. org/10.21037/atm-21-807

Conflicts of Interest: All authors have completed the ICMJE uniform disclosure form (available at http://dx.doi. org/10.21037/atm-21-807). The authors have no conflicts of interest to declare.

Etbical Statement: The authors are accountable for all aspects of the work in ensuring that questions related to the accuracy or integrity of any part of the work are appropriately investigated and resolved. Animal protocols in this study were approved by the Animal Care and Use Committee of First People's Hospital of Shangqiu City. All steps were approved by and conducted following the Guide for the Care and Use of Laboratory Animals.

Open Access Statement: This is an Open Access article distributed in accordance with the Creative Commons Attribution-NonCommercial-NoDerivs 4.0 International License (CC BY-NC-ND 4.0), which permits the noncommercial replication and distribution of the article with the strict proviso that no changes or edits are made and the original work is properly cited (including links to both the formal publication through the relevant DOI and the license). See: https://creativecommons.org/licenses/by-nc-nd/4.0/.

\section{References}

1. Vallabhajosyula S, Dunlay SM, Prasad A, et al. Acute noncardiac organ failure in acute myocardial infarction with cardiogenic shock. J Am Coll Cardiol 2019;73:1781-91.

2. Benjamin EJ, Muntner P, Alonso A, et al. Heart disease and stroke Statistics-2019 update: a report from the American Heart Association. Circulation, 2019;139:e56-e528.

3. Jennings RB. Historical perspective on the pathology of myocardial ischemia/reperfusion injury. Circ Res 2013;113:428-38.

4. Khalid MF, Khan AA, Khattak F, et al. Culprit vessel only 
versus multivessel percutaneous coronary intervention in acute myocardial infarction with cardiogenic shock: a systematic review and meta-analysis. Cardiovasc Revasc Med 2019;20:956-64.

5. Murphy E, Steenbergen C. Mechanisms underlying acute protection from cardiac ischemia- reperfusion injury. Physiol Rev 2008;88:581-609.

6. Chi SW, Zang JB, Mele A, et al. Ago HITS-CLIP decodes miRNA-mRNA interaction maps. Nature 2009;460:479.

7. Chakraborty C, Das S. Profiling cell-free and circulating miRNA: a clinical diagnostic tool for different cancers. Tumour Biol 2016;37:5705-14.

8. Navickas R, Gal D, Laucevi ius A, et al. Identifying circulating microRNAs as biomarkers of cardiovascular disease: a systematic review. Cardiovasc Res 2016;111:322-37.

9. Martino F, Lorenzen J, Schmidt J, et al. Circulating microRNAs are not eliminated by hemodialysis. PLoS One 2012;7:e38269.

10. Pan W, Wang L, Zhang XF, et al. Hypoxia-induced microRNA-191 contributes to hepatic ischemia/ reperfusion injury through the ZONAB/Cyclin D1 axis. Cell Death Differ 2019;26:291-305.

11. Chen Z, Han Y, Deng C, et al. Inflammation-dependent downregulation of miR-194-5p contributes to human intervertebral disc degeneration by targeting CUL4A and CUL4B. J Cell Physiol 2019;234:19977-89.

12. Bus P, Kestens C, Kate FJWT, et al. Profiling of circulating microRNAs in patients with Barrett's esophagus and esophageal adenocarcinoma. J Gastroenterol 2016;51:560-70.

13. Wang M, Li Z, Zuo Q. miR-194-5p inhibits LPS-induced astrocytes activation by directly targeting neurexophilin 1 . Mol Cell Biochem 2020;471:203-13.

14. Livak KJ, Schmittgen TD. Analysis of relative gene expression data using real-time quantitative PCR and the 2(-Delta Delta C(T)) method. Methods 2001;25:402-8.

15. National Research Council (US) Committee. Guide for the Care and Use of Laboratory Animals. National Academies Press (US) 2011; 8th edition.

16. Reichert K, Colantuono B, McCormack I, et al. Murine left anterior descending (lad) coronary artery ligation: an improved and simplified model for myocardial infarction. J Vis $\operatorname{Exp} 2017 ; 2: 55353$.

17. Huang ZQ, Xu W, Wu JL, et al. MicroRNA-374a protects against myocardial ischemia-reperfusion injury in mice by targeting the MAPK6 pathway. Life Sci 2019;232:116619.

18. Lee GH, Jin SW, Kim SJ, et al. Tetrabromobisphenol A induces MMP-9 expression via NADPH oxidase and the activation of ROS, MAPK, and Akt pathways in human breast cancer MCF-7 cells. Toxicol Res 2019;35:93.

19. Cadenas $\mathrm{S}$. ROS and redox signaling in myocardial ischemia-reperfusion injury and cardioprotection. Free Radic Biol Med 2018;117:76-89.

20. Flemming A. Heart failure: targeting miRNA pathology in heart disease, Nat Rev Drug Discov 2014;13:336.

21. Zhou Y, Chen Q, Lew KS, et al. Discovery of potential therapeutic miRNA targets in cardiac ischemia-reperfusion injury. J Cardiovasc Pharmacol Ther 2016;21:296-309.

22. Hilbert T, Markowski P, Frede S, et al. Synthetic CpG oligonucleotides induce a genetic profile ameliorating murine myocardial I/R injury. J Cell Mol Med 2018;22:3397-407.

23. Yaoita H, Ogawa K, Maehara K, et al. Apoptosis in relevant clinical situations:contribution of apoptosis in myocardial infarction. Cardiovasc Res 2000;45:630-41.

24. Wang $X$. The expanding role of mitochondria in apoptosis. Genes Dev 2001;15:2922-33.

25. Granger DN, Kvietys PR. Reperfusion injury and reactive oxygen species: The evolution of a concept. Redox Biol 2015;6:524-51.

26. He F, Zheng G, Hou J, et al. N-acetylcysteine alleviates post-resuscitation myocardial dysfunction and improves survival outcomes via partly inhibiting NLRP3 inflammasome induced-pyroptosis. J Inflamm (Lond) 2020;17:25.

27. Kim EK, Choi EJ. Compromised MAPK signaling in human diseases: an update. Arch Toxicol 2015;89:867-82.

28. Muslin AJ. MAPK signaling in cardiovascular health and disease: molecular mechanisms and therapeutic targets. Clin Sci (Lond) 2008;115:203-18.

29. Kaminska B. MAPK signalling pathways as molecular targets for anti-inflammatory therapy--from molecular mechanisms to therapeutic benefits. Biochim Biophys Acta 2005;1754:253-62.

30. Lee YR, Chen M, Pandolfi PP. The functions and regulation of the PTEN tumour suppressor: new modes and prospects. Nat Rev Mol Cell Biol 2018;19:547-62.

31. Ebbesen SH, Scaltriti M, Bialucha CU, et al. Pten loss promotes MAPK pathway dependency in HER2/ neu breast carcinomas. Proc Natl Acad Sci U S A 2016;113:3030-5.

32. Lu XX, Cao LY, Chen X, et al. PTEN Inhibits Cell Proliferation, Promotes Cell Apoptosis, and Induces Cell Cycle Arrest via Downregulating the PI3K/AKT/hTERT Pathway in Lung Adenocarcinoma A549 Cells. Biomed Res Int 2016;2016:2476842.

33. Liu L, Zhao X, Pierre SV, et al. Association of PI3K-Akt 
signaling pathway with digitalis-induced hypertrophy of cardiac myocytes. Am J Physiol Cell Physiol 2007;293:C1489-C1497.

34. Yu L, Li Z, Dong X, et al. Polydatin Protects Diabetic Heart against Ischemia-Reperfusion Injury via Notch1/ Hes1-Mediated Activation of Pten/Akt Signaling. Oxid

Cite this article as: Zhang Q, Wu X, Yang J. miR-194-5p protects against myocardial ischemia/reperfusion injury via MAPK1/PTEN/AKT pathway. Ann Transl Med 2021;9(8):654. doi: 10.21037/atm-21-807
Med Cell Longev 2018;2018:2750695.

35. Xu J, Tang Y, Bei Y, et al. miR-19b attenuates H2O2induced apoptosis in rat $\mathrm{H} 9 \mathrm{C} 2$ cardiomyocytes via targeting PTEN. Oncotarget 2016;7:10870-8.

(English Language Editor: J. Chapnick) 